\title{
DOENÇA INTERSTICIAL PULMONAR ASSOCIADA À DOENÇA INFLAMATÓRIA INTESTINAL: RELATO DE DOIS CASOS
}

\author{
INTERSTITIAL PULMONARY DISEASE AND INFLAMMATORY \\ BOWEL DISEASE ASSOCIATION: 2 CASES REPORT
}

Lilian Schade ${ }^{1}$, Eliane Ribeiro Carmes², Dante Luiz Escuissato ${ }^{3}$, João Adriano de Barros ${ }^{4}$

\begin{abstract}
${ }^{1}$ Acadêmica de Medicina. ${ }^{2}$ Médica. Departamento de Clínica Médica. ${ }^{3}$ Docente. Departamento de Clínica Médica. ${ }^{4}$ Coordenador do Ambulatório de Doença Pulmonar Intersticial. - Hospital das Clínicas. Universidade Federal do Paraná.

Correspondência: Lilian Schade. Rua Mariano Torres, no 401, apto 403 - Centro. CEP 80060-120 Curitiba - PR.

Email: lilianschade@yahoo.com.br
\end{abstract}

Schade L, Carmes ER, Escuissato DL, Barros JA. Doença intersticial pulmonar associada à doença inflamatória intestinal: Relato de dois casos. Medicina (Ribeirão Preto) 2007; 40 (2): 260-4.

RESUMO: O acometimento pulmonar na doença inflamatória intestinal é raro e não está bem caracterizado. Relatamos os casos de duas pacientes com sintomatologia pulmonar inespecífica, em tratamento prévio de doença inflamatória intestinal - doença de Crohn (caso 1) e retocolite ulcerativa (caso 2). Os achados tomográficos foram compatíveis com pneumonia intersticial usual (PIU). Foi realizada revisão da literatura sobre o assunto.

Descritores: Doenças Pulmonares Intersticiais. Fibrose Pulmonar. Enteropatias Inflamatórias. Doença de Crohn. Proctocolite.

\section{1- INTRODUÇÃO}

A doença inflamatória intestinal está associada a diversas manifestações extra-intestinais. São freqüentes as dermatológicas, reumatológicas, oculares, hepatobiliares e urológicas ${ }^{1} \mathrm{O}$ acometimento pulmonar é raro ${ }^{2,3}$ e não está bem caracterizado ${ }^{1,4}$

Este artigo apresenta o relato de dois casos de doença intersticial pulmonar associada à doença inflamatória intestinal - doença de Crohn $\left(1^{\circ}\right.$ caso) e retocolite ulcerativa idiopática $\left(2^{\circ}\right.$ caso $)$ - e revisão da literatura sobre o assunto.

\section{2- RELATO DOS CASOS}

\section{1- Caso 1}

Paciente feminina, branca, 77 anos, costureira aposentada, natural de Caxias do Sul, RS e residente em Curitiba, PR. Atendida em outubro de 2000 referindo dispnéia aos grandes esforços, tosse com escarro mucoso, 6 episódios/dia de diarréia não purulenta e não sanguinolenta e emagrecimento de $10 \mathrm{~kg}$ nos dois últimos anos. Negava tabagismo e exposição a substâncias de hipersensibilidade. Na ocasião, relatou fibrose pulmonar - diagnosticada em 1993 por meio de biópsia -, doença de Crohn e hipotireoidismo diagnosticados em 1998. Estava em uso de deflazacort 15 $\mathrm{mg} / \mathrm{dia}$, colchicina $1 \mathrm{mg} /$ dia e levotiroxina $100 \mathrm{mcg} / \mathrm{dia}$. Ao exame físico apresentava-se eupneica, afebril e com baqueteamento digital. À ausculta pulmonar crepitantes em 1/3 inferior bilateral. Exame histopatológico, de biópsia transbrônquica realizado em outro serviço, evidenciou proliferação de fibroblastos com depósito de fibras colágenas de distribuição heterogênea no parênquima pulmonar, associado a infiltrado linfocitário em menor intensidade e descamação ma- 
crofágica discreta em alguns alvéolos, compatível com pneumonia intersticial usual (PIU). Colonoscopias realizadas em 1999 e 2000 confirmavam o diagnóstico de doença de Crohn. Foi solicitada radiografia simples de tórax que demonstrou infiltrado com padrão reticular de predomínio basal e periférico (Figura 1), melhor caracterizado à tomografia computadorizada de tórax, em que se observou infiltrado intersticial difuso, caracterizado por espessamento septal com distorção do parênquima pulmonar de aspecto reticular, associado a áreas de faveolamento e bronquioloectasias de tração, predominantes nas regiões periféricas e em lobos inferiores, além de áreas esparsas de consolidação em "vidro fosco" bilateralmente, compatível com PIU. Espirometria de março de 2001 não evidenciou distúrbio ventilatório, os fluxos foram supranormais $\left(\mathrm{VEF}_{1} / \mathrm{CVF}>110 \%\right.$ do previsto $\left.^{5}\right)$, $\mathrm{CVF}=2,41 \mathrm{~L}(85 \%), \mathrm{VEF}_{1}=2,22 \mathrm{~L}(109 \%)$, $\mathrm{FEF}_{25-75 \%}=4,31 \mathrm{~L} / \mathrm{s}(185 \%), \mathrm{VEF}_{1} / \mathrm{CVF}=92 \%$ $(115 \%)$ e prova broncodilatadora negativa. $\mathrm{PaO}_{2}=$ $74 \mathrm{mmHg}$ e $\mathrm{PaCO}_{2}=38,4 \mathrm{mmHg}$. Optou-se, naquele momento, por aumentar a dose de deflazacort para $60 \mathrm{mg} / \mathrm{dia}$ e diminuir gradativamente até $15 \mathrm{mg} / \mathrm{dia}$, as demais medicações foram mantidas. A paciente apresentou melhora do quadro pulmonar e intestinal. Em novembro de 2002 a espirometria demonstrou distúrbio restritivo leve com $\mathrm{CVF}=2,03 \mathrm{~L}(74 \%)$. Em março de 2003, após quadro pulmonar infeccioso, evoluiu com piora da dispnéia. $\mathrm{A} \mathrm{SaO}_{2}$ variou entre 82 e $90 \%$ e houve piora da CVF $=1,92 \mathrm{~L}(71 \%)$. O ecocardiograma revelou hipertensão pulmonar moderada $(\mathrm{PSAP}=51 \mathrm{mmHg})$. A tomografia de tórax (Figura 2) foi semelhante à de 2000. Iniciou ciclofosfamida $100 \mathrm{mg} / \mathrm{dia}$, oxigenioterapia e pulsoterapia semanal com $1 \mathrm{~g}$ de metilprednisolona. Em 2004 evoluiu com piora progressiva da doença e óbito em junho de 2005, por insuficiência respiratória crônica agudizada.

\section{2- Caso 2}

Paciente feminina, branca, 69 anos, do lar, natural de Ponta Grossa-PR e residente em Curitiba- PR. Consultou em agosto de 2003 com queixas de dispnéia aos grandes esforços, tosse seca ocasional, diarréia mucosa, 3 a 5 episódios/dia e dor ab-

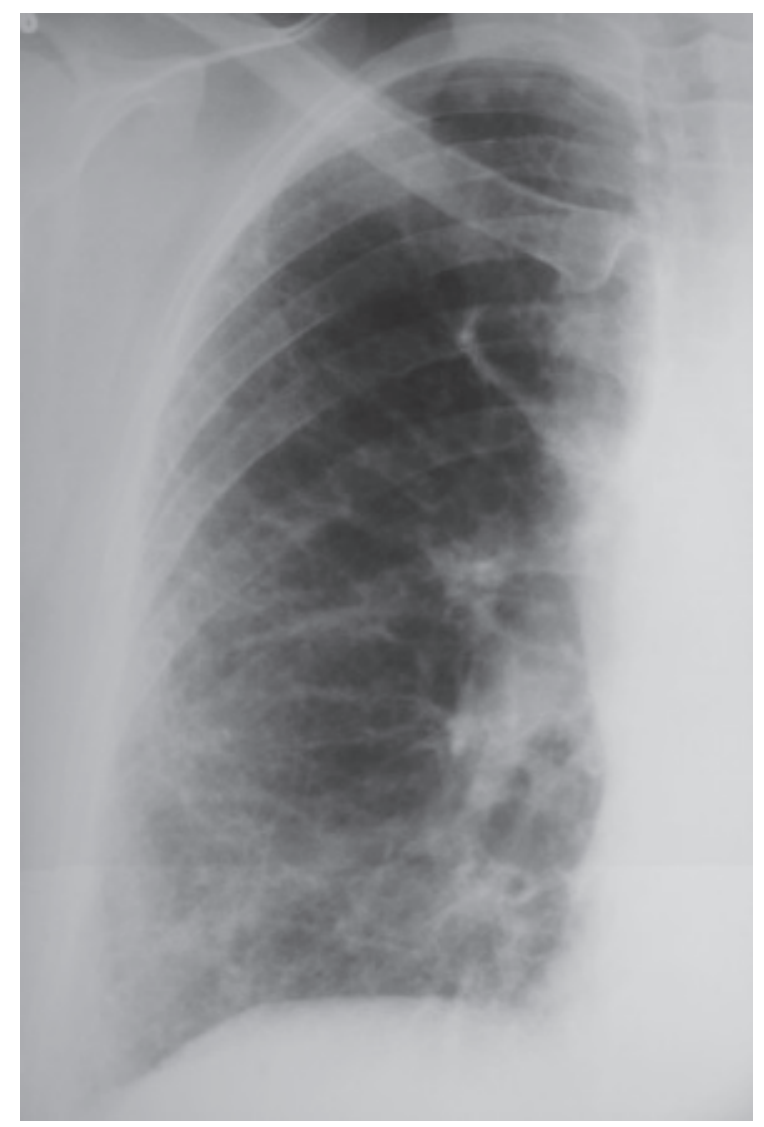

Figura 1: Radiografia frontal de hemitórax direito mostra infiltrado com padrão reticular e predomínio basal e periférico.

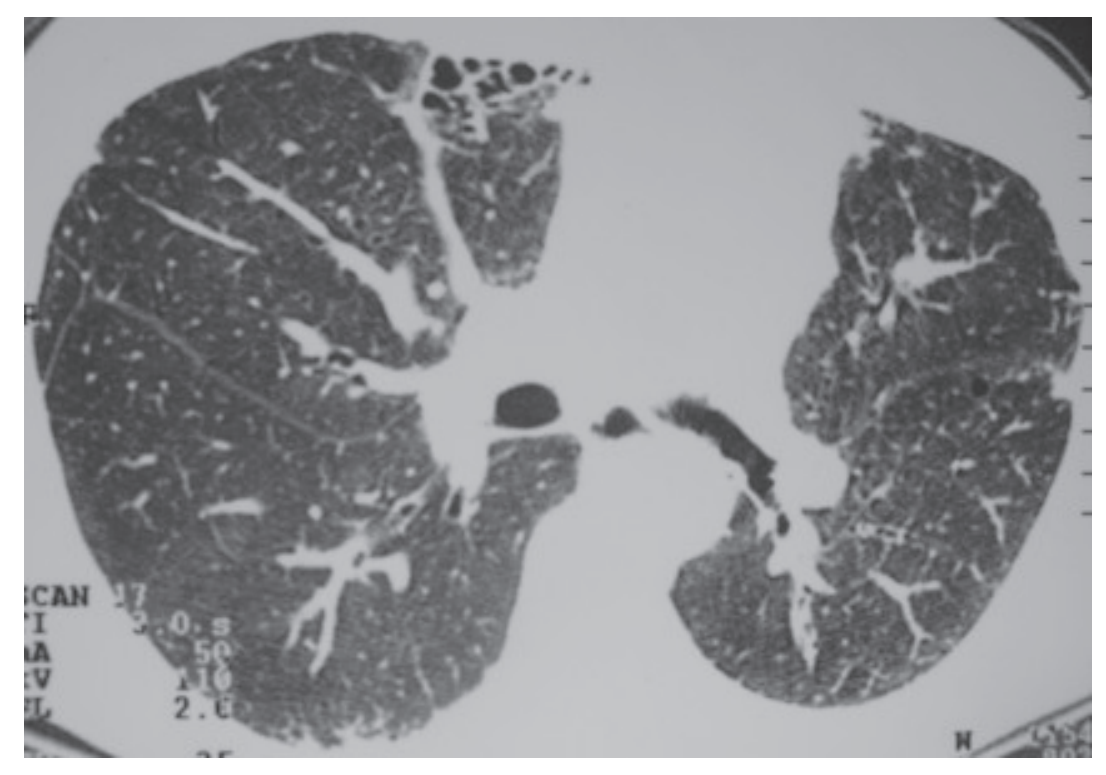

Figura 2: Os cortes de tomografia computadorizada de alta resolução, obtidos ao nível dos hilos pulmonares, mostram infiltrados pulmonares de aspecto reticular com áreas de faveolamento. Focos de atenuação em vidro fosco são observados associados a estas lesões. 
dominal esporádica e difusa. Não tabagista, com antecedentes de doença intersticial pulmonar diagnosticada em outubro de 2002. Apresentava como comorbidades hipertensão, hipotireoidismo, fibromialgia e artralgia crônica, periférica e assimétrica, sem sinais inflamatórios, deformidades ou impotência funcional. Ao exame físico apresentava-se eupneica e afebril, com baqueteamento digital e crepitantes em bases pulmonares. Exames laboratoriais e de imagem haviam sido realizados em outro serviço em outubro e novembro de 2002. A tomografia computadorizada de tórax evidenciava espessamento de septos pulmonares com bronquioloectasias de tração, atelectasias subsegmentares e áreas de faveolamento, compatíveis com PIU. À colonoscopia e retossigmoidoscopia foi observado pancolite enantemática e retocolite ulcerativa com atividade leve. Esta confirmada por exame histopatológico da mucosa colônica, que apresentava padrão arquitetural conservado, discreto espessamento de membrana basal e moderado infiltrado linfoplasmocitário. Proteína C reativa 30,8, FAN 1:320, auto-anticorpos (anti-DNA, anti-SCL, anti-centômero, anti-RNP, antiRo, anti-SM) não reagentes; C3, C4 e eletroforese de proteínas nos limites da normalidade. Na ocasião da consulta foram solicitadas radiografia de tórax e tomografia de tórax (Figura 3) que foram compatíveis com PIU. Prova de função pulmonar demonstrou redução isolada de fluxos expiratórios, $\mathrm{CVF}=2,19 \mathrm{~L}$ $(87 \%), \mathrm{VEF}_{1}=1,65 \mathrm{~L}(82 \%), \mathrm{FEF}_{25-}$ $75 \%=1,30 \mathrm{~L}(46 \%), \mathrm{VEF}_{1} / \mathrm{CVF}=75 \%$ (91\%), prova broncodilatadora negativa e redução isolada da capacidade inspiratória: CI 0,99L (49\%). A oximetria de pulso em repouso foi normal $(97 \%)$. Exames laboratoriais: fator reumatóide negativo $(<10,3)$ e VHS 40mm em 1 hora. Tem-se mantida clinicamente estável com sulfassalazina $2 \mathrm{~g} /$ dia, prednisona $7,5 \mathrm{mg} /$ dia, levotiroxina $100 \mathrm{mg} /$ dia, amitriptilina $25 \mathrm{mg} /$ dia, captopril $150 \mathrm{mg} / \mathrm{dia}$, hidroclorotiazida $25 \mathrm{mg} /$ dia e nifedipina $40 \mathrm{mg} /$ dia.

\section{3- DISCUSSÃO}

As doenças intersticiais pulmonares representam um conjunto de doenças que acometem o parênquima pulmonar causando inflamação e fibrose. Algumas com etiologia co- nhecida, tais como fármacos, exposição ambiental (fumaças, poeiras, penas e excrementos de aves), exposição ocupacional (sílica, asbesto, carvão, entre outros) e colagenoses (lupus sistêmico, artrite reumatóide, esclerose sistêmica). Para outras a etiologia é desconhecida, são exemplos a fibrose pulmonar idiopática e a sarcoidose ${ }^{6}$. O diagnóstico depende de características clínicas, radiológicas e histopatológicas. A radiografia de tórax é inespecífica e isoladamente não permite o diagnóstico. A tomografia computadorizada de tórax possibilita uma melhor avaliação da distribuição e extensão do comprometimento pulmonar A biópsia é considerada o "padrão ouro" para o diagnóstico da doença. Ainda assim, tem indicação discutida face o grau de resolução atual das imagens obtidas por tomografia computadorizada que possibilitam tipificar alguns padrões de doença. Imagens essas, que associadas com o quadro clínico do paciente, podem muitas vezes tornar desnecessário o procedimento cirúrgico para confirmar o diagnóstico ${ }^{7 / 10}$, é o que ocorre na PIU e nas condições de padrão muito específico. Na PIU a biópsia pode ser desnecessária quando na presença de tomografia computadorizada de tórax altamente sugestiva ${ }^{9}$. As provas de função pulmonar indicam a gravidade da doença. Normalmente são encontrados distúrbios ventilatórios restritivos e redução da capacidade de difusão do monóxido de carbono $^{11}$.

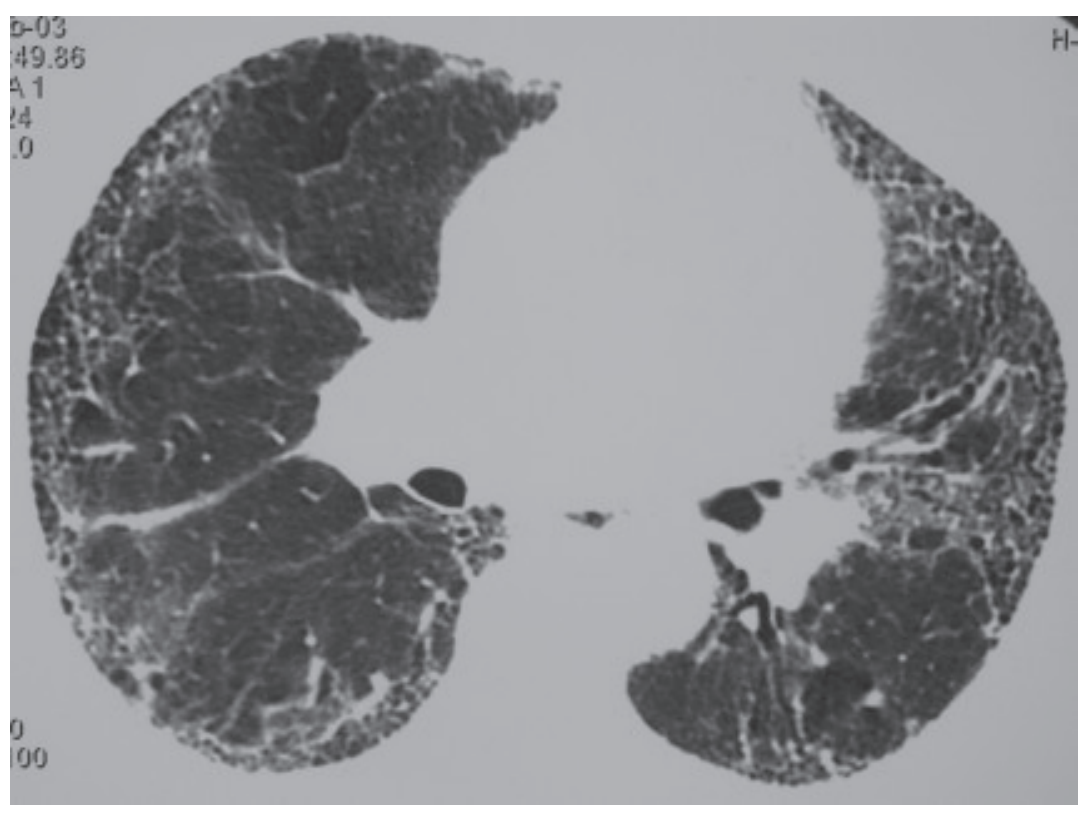

Figura 3: Imagem de tomografia computadorizada de alta resolução dos terços médios dos pulmões mostra infiltrados reticulares periféricos de distribuição assimétrica com áreas de faveolamento associadas. 
Nos casos acima relatados o diagnóstico de doença intersticial pulmonar baseou-se nas manifestações clínicas, radiológicas, histopatolágicas e provas de função pulmonar. Não se encontraram evidências de possíveis fatores etiológicos para os casos acima descritos, mas observou-se a associação com doença inflamatória intestinal, já descrita na literatura.

$\mathrm{O}$ acometimento pulmonar na doença inflamatória intestinal é raro ${ }^{2,3}$ e sua prevalência é desconhecida $^{4}$ Em uma breve revisão da literatura encontramos relatos de doença pulmonar associada à doença de Crohn e retocolite ulcerativa. Entre 1996 e 1998 Kuzela et. al. ${ }^{18}$ investigaram a ocorrência de doença pulmonar com doença inflamatória intestinal, estudando 82 pacientes ( 30 pacientes com colite ulcerativa e 52 com doença de Crohn) e um grupo controle de 60 indivíduos. Dentre os parâmetros utilizados (manifestações clínicas, radiografia de tórax e provas de função pulmonar) houve diferença significativa na função pulmonar dos dois grupos ( $\mathrm{p}<0,001): 56,7 \%$ dos pacientes com doença de Crohn e $57,7 \%$ dos pacientes com colite ulcerativa apresentavam capacidade de difusão reduzida.

A patogênese é ainda desconhecida. Foram descritos casos de progressão da doença pulmonar até mesmo após colectomia ${ }^{13,15}$. Os relatos abrangem uma ampla faixa etária, não há predominância entre os sexos, e, antecedentes de tabagismo não são verificados na grande maioria dos $\operatorname{casos}^{1 / 4,12 / 17}$.

Os sintomas pulmonares ocorrem na maioria das vezes após o diagnóstico da doença intestinal, mas pode preceder (como no caso 1) ou ser concomitante ao diagnóstico (caso 2) ) $^{1,13}$. Tem-se observado um intervalo de tempo médio menor que um ano quando o acometimento pulmonar precede o intestinal, embora haja relato de um intervalo de até oito anos ${ }^{16}$. O tempo decorrido de acometimento pulmonar após o intestinal é bastante variável $(4-192 \text { meses })^{1}$.

As manifestações clinicas, radiológicas e anatomopatológicas da doença pulmonar na doença inflamatória intestinal não estão bem caracterizadas ${ }^{1,4} \mathrm{Na}$ doença de Crohn, a maioria dos pacientes apresenta infiltrados pulmonares difusos incluindo bronquiolite difusa, bronquiolite obliterante com pneumonia em organização (BOOP) e pneumonia intersticial. BOOP é o achado mais freqüente. Menos comumente são encontrados infiltrados localizados, que correspondem geralmente a uma combinação de BOOP e inflamação granulomatosa ${ }^{1,4,19} \mathrm{Na}$ retocolite ulcerativa é mais freqüente o acometimento de via aérea, sendo bronquite e bronquiectasia os achados mais comuns, embora acometimentos intersticial, vascular e pleural também sejam descritos ${ }^{16,17}$. Os dois casos em discussão apresentam imagens compatíveis com PIU, confirmada por exame histopatológico no caso 1 . O caso 2 exemplifica a situação em que a imagem tomográfica típica dentro de um contexto clínico adequado torna o diagnóstico possível sem a realização de biópsia.

Ressalta-se, por fim, que o acometimento pulmonar na doença inflamatória intestinal é relevante e, pela raridade dos relatos, ainda pouco caracterizado.

Schade L, Carmes ER, Escuissato DL, Barros JA. Interstitial pulmonary disease and inflammatory bowel disease association: 2 Cases Report. Medicina (Ribeirão Preto) 2007; 40 (2): 260-4.

ABSTRACT: The lung involvement in the inflammatory bowel disease is rare and it is not well characterized. We describe two cases of female patients with unspecific pulmonary symptomatology, and previous treatment for inflammatory bowel disease - Crohn's disease (case 1 ) and ulcerative rectocolitis. A chest computerized tomography was compatible with usual interstitial pneumonia (UIP). Related literature was reviewed.

Keywords: Lung Diseases, Interstitial. Pulmonary Fibrosis. Inflammatory Bowel Diseases. Crohn Disease. Proctocolitis. 


\section{REFERÊNCIAS}

1 - Casey MB, Tazelaar HD, Myers JL, Hunninghake GW, Kakar $S$, Kalra SX et al. Noninfectious lung pathology in patients with Crohn's disease. Am J Surg Pathol 2003; 27(2): 213-9.

2 - Dawson A, Gibbs AR, Anderson G. An unusual perilobular pattern of pulmonary interstitial fibrosis associated with Cronh's disease. Histopathology 1993; 23 (6): 553-6.

3 - Omori H, Asahi H, Inoue Y, Irinoda T, Saito K. Pulmonary involvement in Cronh's disease report of a case and review of the literature. Inflamm Bowel Dis 2004; 10(2): 129-34.

4 - Mahadeva R, Walsh G, Flower CD, Shneerson JM. Clinical and radiologial caracteristics of lung disease in inflamatory bowel disease. Eur Respir J 2000; 15(1):41-8.

5 - Diretrizes para teste de função pulmonar. J Pneumol 2002; 28(3): S58-S67.

6 - American Thoracic Society. ATS/ERS international consensus classification of idiopathic interstitial pneumonias. Am J Respir Crit Care Med 2002; 165(2): 277-304.

7 - Capelozzi VL. Dificuldade na interpretação de biopsias em doenças pulmonares difusas. J Pneumol 1998; 24(1): 3042.

8 - Webb WR, Müller NL, Naiolich DP. High-resolution CT of the lung. 2 ed. New York: Lippincott - Raven; 1996: 271-91. Clinical utility of the HRCT and indications for its use.

9 - Diette GB, Scatarige JC, Haponik EF, Merriman B, Fishman EK. Do high-resolution CT findings of usual interstitial pneumonitis obviate lung biopsy? Respiration 2005; 72(2): 13441.

10 - Swensen SJ, Aughenbaugh GL, Myers JL. Diffuse lung disease: diagnostic accuracy of CT in patients undergoing surgical biopsy of the lung. Radiology 1997; 205(1): 229-34.
11 - Boros PW, Franczuk M, Wesolowski S. Value of spirometry in detecting volume restriction in interstitial lung disease patients. Spirometry in interstitial lung disease. Respiration 2004; 71(4): 374-9.

12 - Case records of the Massachusetts General Hospital. Weekly clinicopathological exercises. Case 12-1993. A 44-year-old man with pulmonary disease and proctitis. $N$ Engl $\mathrm{J}$ Med 1993; 328(12): 869-76.

13 - Camus P, Piard F, Ashcroft T, Gal AA, Colby TV. The lung in inflamatory bowel disease. Medicine (Baltimore) 1993; 72(3): 151-83.

14 - Chikano S, Sawada K, Ohnishi K, Fukunaga K, Tanaka J, Shimoyama T. Interstitial pneumonia accompanying ulcerative colitis. Intern Med 2001; 40(9): 883-6.

15 - Eaton TE, Lambie N, Wells AU. Bronchiectasis following colectomy for Crohn's disease. Thorax 1998; 53: 529-31.

16 - Desai SJ, Gephardt GN, Stoller JK. Diffuse panbronchiolitis preceding ulcerative colitis. Chest 1989; 45: 1342-4.

17 - Cohen M, Sahn SA. Bronchiectasis in systemic diseases. Chest 1999; 116:1063-74.

18 - Kuzela L, Vavrecka A, Prikazska M, Drugda B, Hronec J, Senkova AM et al. Pulmorary complication in patients with inflamatory bowel disease. Hepatogastroenterology 1999; 46(27): 1714-9.

19 - Fenoglio-Preiser CM, editors. Gastrointestinal pathology. 2a. ed. New York: Lippincott - Raven; 1999. p.631-716. Inflammatory bowel disease.

Recebido em 06/11/2006

Aprovado em 19/04/2006 